\title{
Introduction to the Physics of Organic Semiconductors
}

\author{
Wolfgang Brütting* \\ Institute of Physics, University of Augsburg, 86135 Augsburg, Germany \\ Received zzz, revised zzz, accepted zzz \\ Published online zzz
}

PACS 00.00.Xx, 11.11.Yy

This article gives a brief introduction to main the scope of this book, which is to provide an up-to-date overview of the different aspects in the physics of organic semiconductors and their application in devices. After a short historical review, some prototype materials will be presented. This will be followed by a discussion of the basic processes in organic semiconductors together with an introduction to the most important device structures. Finally a short outline of this book will be given.

(C) 2003 WILEY-VCH Verlag GmbH \& Co. KGaA, Weinheim

\section{History}

With the invention of the transistor around the middle of the last century, inorganic semiconductors like $\mathrm{Si}$ or Ge began to take over the role as dominant material in electronics from the before prevailing metals. At the same time, the replacement of vacuum tube based electronics by solid state devices initiated a development which by the end of the $20^{\text {th }}$ century has lead to the omnipresence of semiconductor microelectronics in our everyday life. Now at the beginning of the $21^{\text {st }}$ century we are facing a new electronics revolution that has become possible due to the development and understanding of a new class of materials, commonly known as Organic Semiconductors. The enormous progress in this field has been driven by the expectation to realize new applications, such as large area, flexible light sources and displays, low-cost printed integrated circuits or plastic solar cells from these materials.

Strictly speaking organic semiconductors are not new. The first studies of the dark and photoconductivity of anthracene crystals (a prototype organic semiconductor, see e.g. [1]) date back to the early $20^{\text {th }}$ century $[2,3]$. Later on, triggered by the discovery of electroluminescence in the 1960s [4,5], molecular crystals were intensely investigated by many researchers. These investigations could establish the basic processes involved in optical excitation and charge carrier transport (for a review see e.g. [6,7]). Nevertheless, in spite of the principal demonstration of an organic electroluminescent diode incorporating even an encapsulation similar to the ones used in nowadays commercial display applications [8], there were several draw-backs preventing practical use of these early devices. For example, neither high enough current densities and light output nor sufficient stability could be achieved. The main obstacles were the high operating voltage as a consequence of the crystal thickness in the micrometre to millimetre range together with the difficulties in scaling up crystal growth as well as preparing stable and sufficiently wellinjecting contacts to them.

Since the 1970s the successful synthesis and controlled doping of conjugated polymers [9] established the second important class of organic semiconductors which was honoured with the Nobel Prize in Chemistry in the year 2000. Together with organic photoconductors (molecularly doped polymers) these conducting polymers have initiated the first applications of organic materials as conductive coatings [10] or photoreceptors in electrophotography [11]. 
The interest in undoped organic semiconductors revived in the 1980s due to the demonstration of an efficient photovoltaic cell incorporating an organic hetero-junction of $\mathrm{p}$ - and n-conducting materials [12] as well as the first successful fabrication of thin film transistors from conjugated polymers and oligomers [13-15]. The main impetus, however, came from the demonstration of high-performance electroluminescent diodes from vacuum-evaporated molecular films [16,17] and from conjugated polymers [18,19]. Owing to the large efforts of both academic and industrial research laboratories during the last 15 years, organic light-emitting devices (OLEDs) have progressed rapidly and meanwhile lead to first commercial products incorporating OLED displays [20]. Other applications of organic semiconductors e.g. as logic circuits with organic field-effect transistors (OFETs) or organic photovoltaic cells (OPVCs) are expected to follow in the near future (for an overview see e.g. [21]).

\section{Materials}

As already mentioned above, there are two major classes of organic semiconductors: low molecular weight materials and polymers. Both have in common a conjugated $\pi$-electron system being formed by the $\mathrm{p}_{\mathrm{z}}$-orbitals of $\mathrm{sp}^{2}$-hybridized $\mathrm{C}$-atoms in the molecules (see Fig. 1). As compared to the $\sigma$-bonds forming the backbone of the molecules, the $\pi$-bonding is significantly weaker. Therefore, the lowest electronic excitations of conjugated molecules are the $\pi$ - $\pi^{*}$-transitions with an energy gap typically between 1.5 and $3 \mathrm{eV}$ leading to light absorption or emission in the visible spectral range. As shown in Table 1 for the family of the polyacenes the energy gap can be controlled by the degree of conjugation in a molecule. Thus chemistry offers a wide range of possibilities to tune the optoelectronic properties of organic semiconducting materials. Some prototype materials which are also discussed in this book are given in Fig. 2.
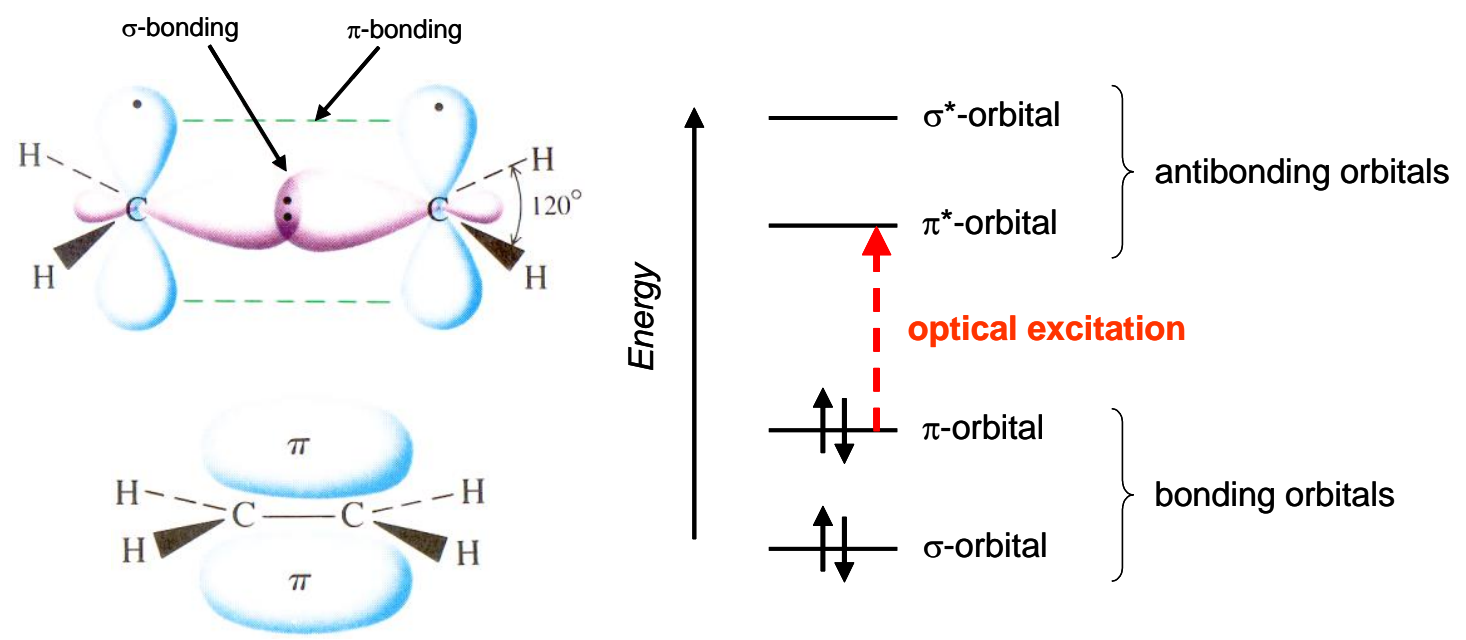

Fig. 1 Left: $\sigma$ - and $\pi$-bonds in ethane, as an example for the simplest conjugated $\pi$-electron system. The right viewgraph shows the energy levels of a $\pi$-conjugated molecule. The lowest electronic excitation is between the bonding $\pi$-orbital and the antibonding $\pi *$-orbital (adopted from [22]).

An important difference between the two classes of materials lies in the way how they are processed to form thin films. Whereas small molecules are usually deposited from the gas phase by sublimation or evaporation, conjugated polymers can only be processed from solution e.g. by spin-coating or printing techniques. Additionally, a number of low-molecular materials can be grown as single crystals allowing intrinsic electronic properties to be studied on such model systems (see e.g. [23] or [24] for an overview of the different classes of materials). The controlled growth of highly ordered thin films either by vacu- 
um deposition or solution processing is still subject of ongoing research, but will be crucial for many applications (see e.g. [25]).

Table 1 Molecular structure of the first five polyacenes, together with the wavelength of the main absorption peak (taken from [7]).

$\begin{array}{|cc|}\text { Molecule } & \begin{array}{c}\text { Absorption } \\ \text { Maximum }\end{array} \\ \text { Benzene } & 255 \mathrm{~nm} \\ \text { Netracene } & 380 \mathrm{~nm} \\ \text { Pentacene } & 580 \mathrm{~nm} \\ 580 \mathrm{~nm}\end{array}$

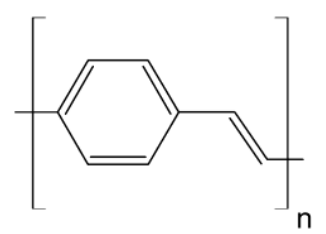

PPV

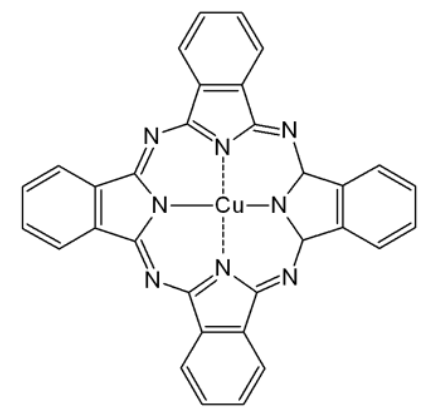

CuPc

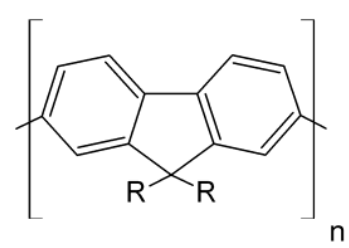

PFO

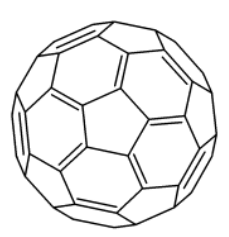

$\mathrm{C}_{60}$

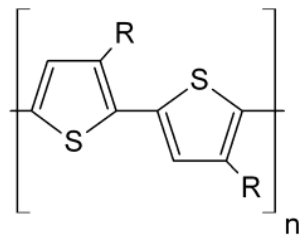

P3AT

Fig. 2 Molecular structure of some prototype organic semiconductors: PPV: poly(p-phenylenevinylene), PFO polyfluorene, P3AT: poly(3-alkylthiophene), $\mathrm{Alq}_{3}$ : tris(8-hydroxyquinoline)aluminium, fullerene $\mathrm{C}_{60}, \mathrm{CuPc}$ : Cu-phthalocyanine, pentacene.

\section{Basic Properties of Organic Semiconductors}

The nature of bonding in organic semiconductors is fundamentally different from their inorganic counterparts. Organic molecular crystals are van der Waals bonded solids implying a considerably weaker intermolecular bonding as compared to covalently bonded semiconductors like Si or GaAs. The conse- 
quences are seen in mechanical and thermodynamic properties like reduced hardness or lower melting point, but even more importantly in a much weaker delocalization of electronic wavefunctions among neighbouring molecules, which has direct implications for optical properties and charge carrier transport. The situation in polymers is somewhat different since the morphology of polymer chains can lead to improved mechanical properties. Nevertheless, the electronic interaction between adjacent chains is usually also quite weak in this class of materials.

\subsection{Optical Properties}

Owing to the weak electronic delocalization, to first order the optical absorption and luminescence spectra of organic molecular solids are very similar to the spectra in the gas phase or in solution (apart from the trivial solvent shift). In particular, intramolecular vibrations play an important role in solid state spectra and often these vibronic modes can be resolved even at room temperature. Thus the term "oriented gas" is sometimes used for molecular crystals. Nevertheless, solid state spectra can differ in detail with respect to selection rules, oscillator strength and energetic position; moreover, due to the crystal structure or the packing of polymer chains a pronounced anisotropy can be found. Additionally disordered organic solids usually show a considerable spectral broadening. This is schematically shown in Fig. 3.

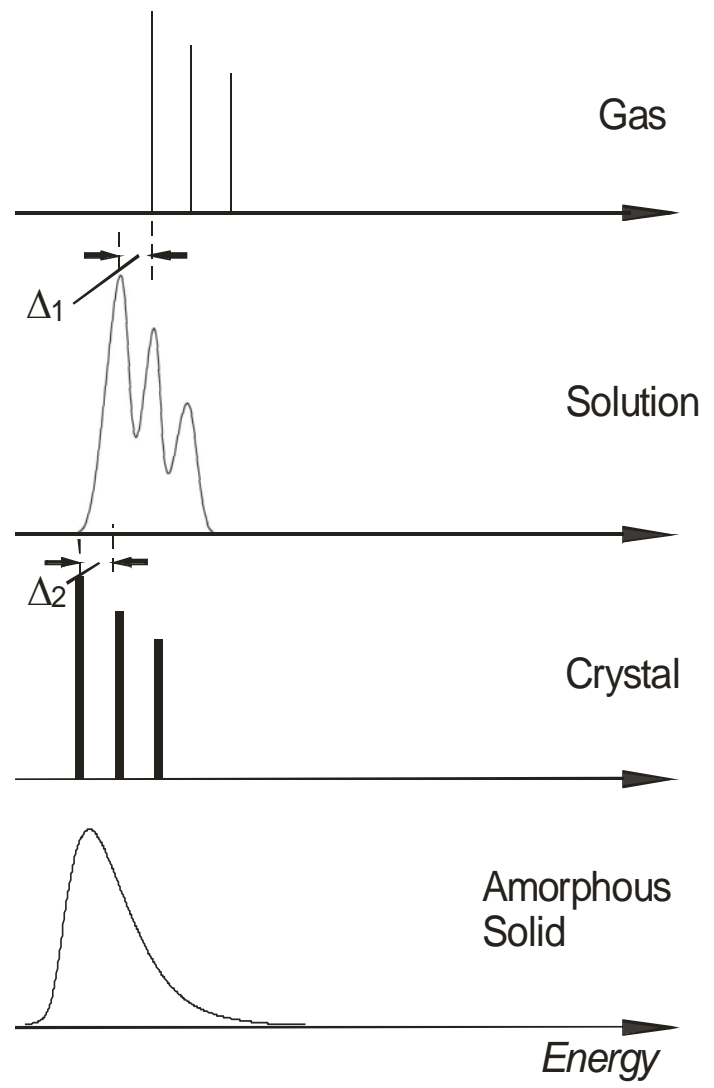

Fig. 3 Schematical representation of optical spectra of organic molecules in different surroundings. $\Delta_{1}$ and $\Delta_{2}$ denote the respective solvent shift in solution and solid state. 


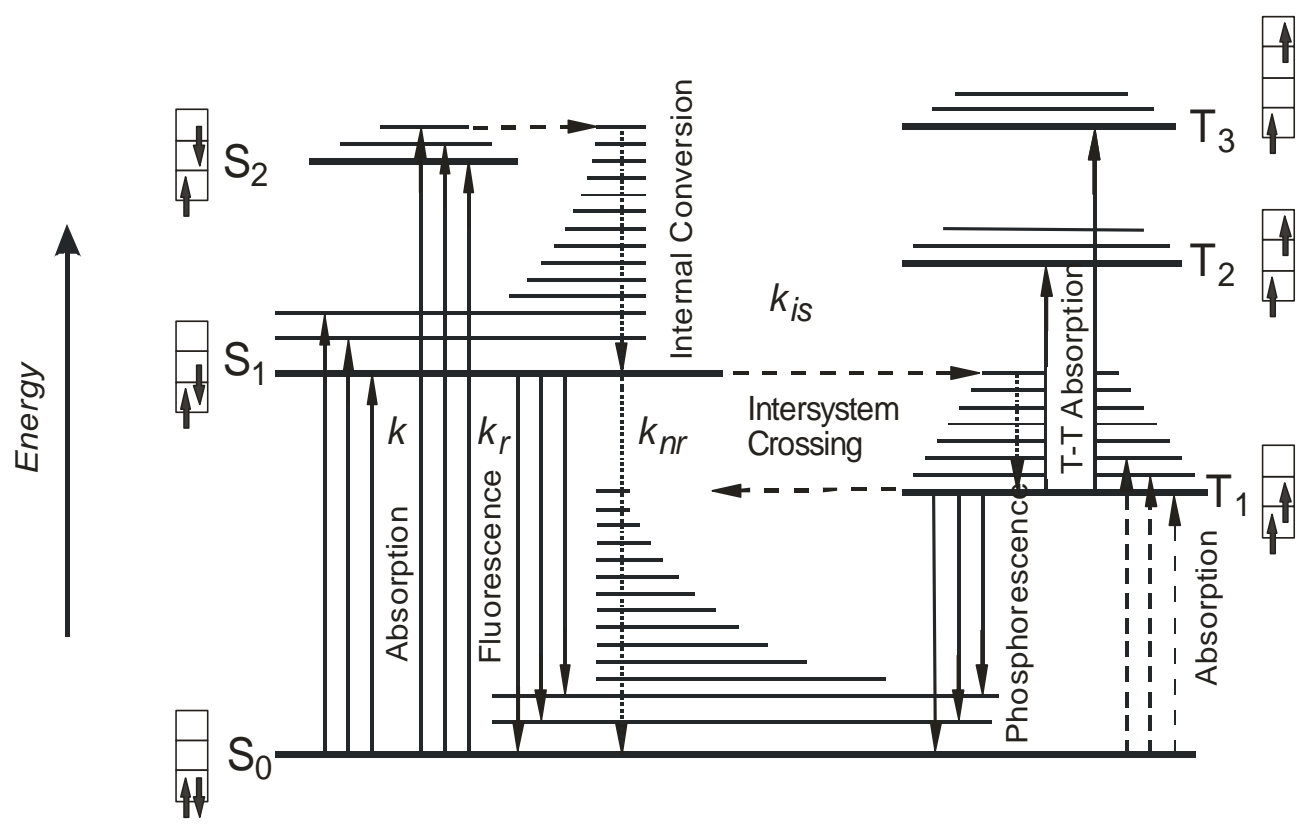

Fig. 4 Energy level scheme of an organic molecule (left: singlet manifold, right: triplet manifold). Arrows with solid lines indicate radiative transitions, those with broken lines nonradiative transitions (taken from [7]). Typical lifetimes of the $S_{1}$ state are in the range $1 \ldots 10 \mathrm{~ns}$. Triplet lifetimes are usually in the millisecond range for pure aromatic hydrocarbons, but can be considerably shorter in molecules incorporating heavy atoms, like e.g. Pt or Ir.

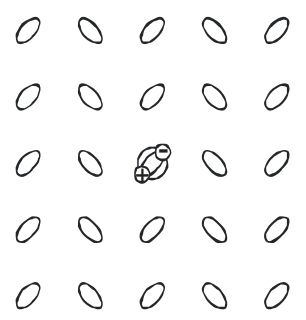

Frenkel Exciton

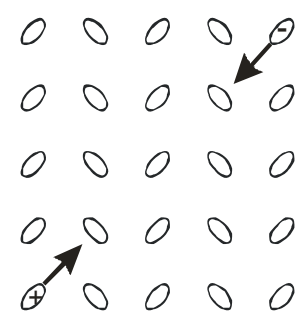

Electron-Hole Recombination/ Exciton Dissociation

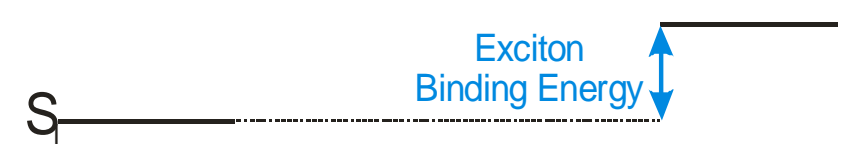

$$
\text { So- }
$$

Fig. 5 The energetic difference between an excited state sitting on one molecule (sometimes called a Frenkel exciton) and a pair of uncorrelated negative and positive carriers sitting on different molecules far apart defines the exciton binding energy. A simple estimation as the Coulomb energy of an electron-hole pair localized at a distance of about $10 \AA$ in a medium with a dielectric constant of 3 yields a value of about $0.5 \mathrm{eV}$ for the exciton binding energy. 
As a consequence of this weak electronic delocalization, organic semiconductors have two important peculiarities as compared to their inorganic counterparts. One is the existence of well-defined spin states (singlet and triplet) like in isolated molecules which has important consequences for the photophysics of these materials (see Fig. 4). However, since intersystem crossing is a weak process, this also sets an upper limit for the electroluminescence quantum efficiency in OLEDs. A second important difference originates from the fact that optical excitations ("excitons") are usually localized on one molecule and therefore have a considerable binding energy of typically 0.5 to $1 \mathrm{eV}$. Thus in a photovoltaic cells this binding energy has to be overcome before a pair of independent positive and negative charge carriers is generated (see Fig. 5).

\subsection{Charge Carrier Transport}

When transport of electrons or holes in an organic molecular solid is considered, one has to bear in mind that this involves ionic molecular states. E.g. in order to create a hole, an electron has to be removed to form a radical cation $\mathrm{M}^{+}$out of a neutral molecule $\mathrm{M}$. This defect electron can then move from one molecule to the next. In the same way, electron transport involves negatively charged radical ions $\mathrm{M}^{-}$. (Qualitatively, the same arguments hold for polymers, however, in this case charged states are usually termed positive or negative polarons.) As compared to isolated molecules in the gas phase, these ionic states are stabilized in the solid by polarization energies leading to an energy level scheme as shown in Fig. 6. From this picture one can clearly see that due to the already mentioned exciton binding energy the optical gap between the ground state and the first excited singlet state is considerably less than the single particle gap to create an uncorrelated electron-hole pair. In going from molecular crystals to disordered organic solids one also has to consider locally varying polarization energies due to different molecular environments which lead to a Gaussian density of states for the distribution of transport sites as shown in Fig. 7.

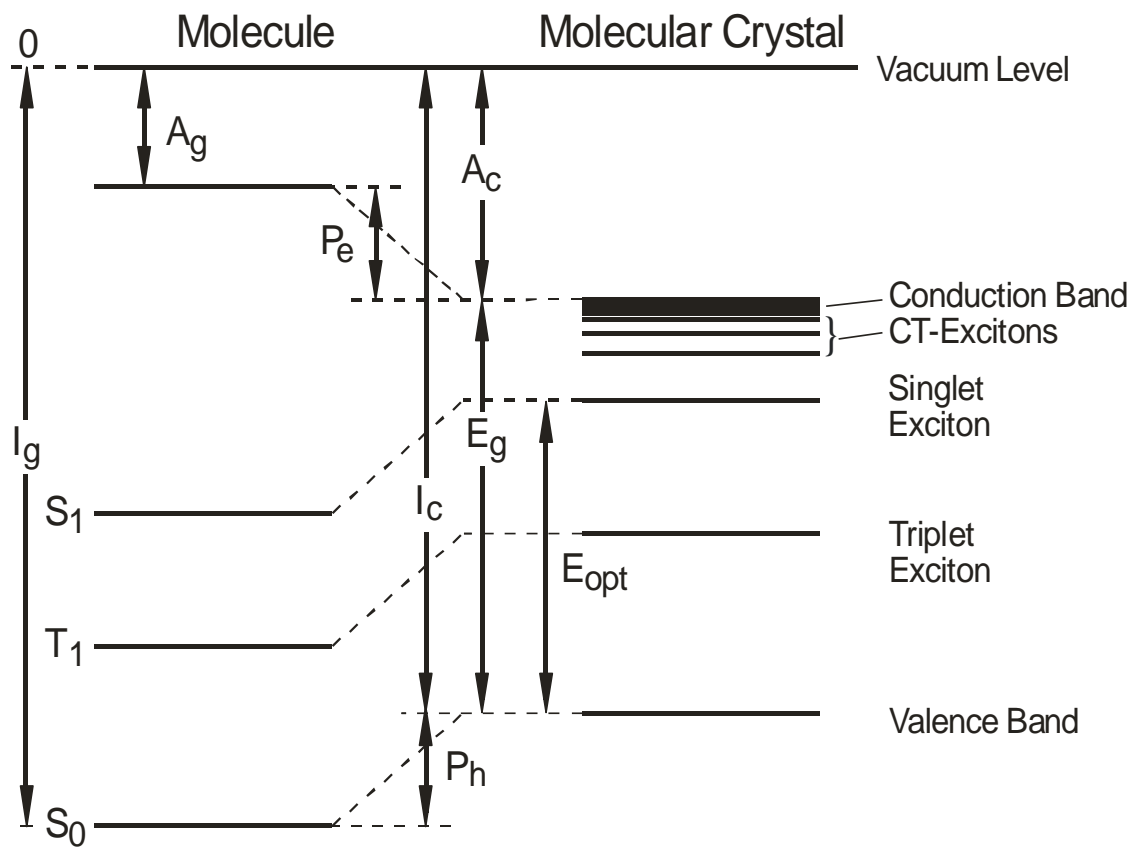

Fig. 6 Energy levels of an isolated molecule (left) and a molecular crystal (right). $I_{g}$ and $A_{g}$ denote the ionization potential and electron affinity in the gas phase, $I_{c}$ and $A_{c}$ the respective quantites in the crystal. Due to the polarization energies $\mathrm{P}_{\mathrm{h}}$ and $\mathrm{Pe}_{\mathrm{e}}$ charged states are stabilized in the crystal. $\mathrm{E}_{\mathrm{g}}$ is the single particle gap being relevant for charge carrier generation, whereas Eopt denotes the optical gap measured in absorption and luminescence. Their difference is the so-called exciton binding energy. (Figure adopted from [7]). 


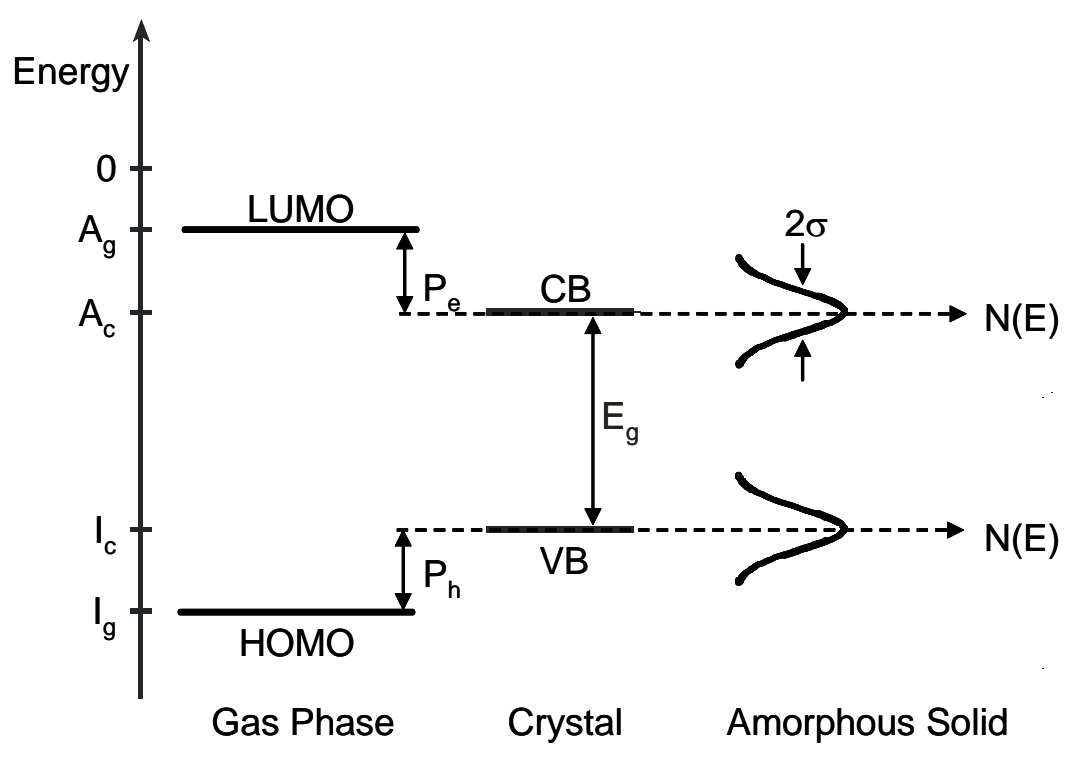

Fig. 7 Energy levels of an isolated molecule (left), a molecular crystal (middle) and an amorphous solid (right). The width of the Gaussian density of states in an amorphous solid is typically in the range of $\sigma=80 \ldots 120 \mathrm{meV}$, whereas the band width in molecular crystals is less than $100 \mathrm{meV}$. (Figure adopted from [26]).

Thus, depending on the degree of order the charge carrier transport mechanism in organic semiconductors can fall between two extreme cases: band or hopping transport. Band transport is typically observed in highly purified molecular crystals at not too high temperatures. However, since electronic delocalization is weak the bandwidth is only small as compared to inorganic semiconductors (typically a few kT at room temperature only). Therefore room temperature mobilities in molecular crystals reach only values in the range 1 to $10 \mathrm{~cm}^{2} / \mathrm{Vs}$ [27]. As a characteristic feature of band transport the temperature dependence follows a power law behaviour

$$
\mu \propto T^{-n} \quad \text { with } \quad n=1 \ldots 3
$$

upon going to lower temperature. However, in the presence of traps significant deviations from such a behaviour are observed [28].

In the other extreme case of an amorphous organic solid hopping transport prevails which leads to much lower mobility values (at best around $10^{-3} \mathrm{~cm}^{2} / \mathrm{Vs}$, in many cases however much less). Instead of a power law the temperature dependence then shows an activated behaviour and the mobility also depends on the applied electric field:

$$
\mu(F, T) \propto \exp (-\Delta E / k T) \cdot \exp (\beta \sqrt{F} / k T)
$$

Depending on the model slightly different temperature dependencies for the mobility have been suggested (for a review see [11]). Furthermore, space-charge and trapping effects as well as details of the charge carrier injection mechanism have to be considered for describing electrical transport in organic solids [29-33].

On a macroscopic level, the current through a material is given by the charge carrier density $n$ and the carrier drift velocity $v$, where the latter can be expressed by the mobility $\mu$ and the electric field $F$ :

$$
j=e n v=e n \mu F
$$


One has to bear in mind that in contrast to metals this is usually not a linear relation between $j$ and $F$ since both the carrier density and mobility can depend on the applied field. According to this equation, apart from the field, the two parameters $n$ and $\mu$ determine the magnitude of the current. Thus it is instructive to compare their typical values with inorganic semiconductors and discuss different ways to control them.

As already discussed above, the mobility strongly depends on the degree of order and purity in organic semiconductors and therefore to a great deal on the preparation and growth conditions. It can reach values of $1-10 \mathrm{~cm}^{2} / \mathrm{Vs}$ in molecular crystals (see the contribution of de Boer et al. in this book), but values of only $10^{-5} \mathrm{~cm}^{2} / \mathrm{Vs}$ in amorphous materials are also not unusual. The highest mobility values achievable in thin films are nowadays comparable to amorphous silicon which is of course orders of magnitude less than crystalline Si [34-36].

The second parameter is the charge carrier density $n$. The intrinsic carrier density in a semiconductor with an energy gap $E_{g}$ and an effective density of states $N_{0}$ (which is strictly speaking the product of valence and conduction band densities) is given by:

$$
n_{i}=N_{0} \cdot \exp \left(-E_{g} / 2 k T\right)
$$

Taking typical values for an organic semiconductor with $E_{g}=2.5 \mathrm{eV}$ and $N_{0}=10^{21} \mathrm{~cm}^{-3}$ leads to a hypothetical carrier density of $n_{i}=1 \mathrm{~cm}^{-3}$ at room temperature, which is of course never reachable since impurities will lead to much higher densities in real materials. Nevertheless, the corresponding value for $\mathrm{Si}$ $\left(E_{g}=1.12 \mathrm{eV}\right.$ and $\left.N_{0}=10^{19} \mathrm{~cm}^{-3}\right)$ is with $n_{i}=10^{10} \mathrm{~cm}^{-3}$ many orders of magnitude higher, which demonstrates that organic semiconductors should have extremely low conductivity if they are pure enough. In order to overcome the limitations posed by the low intrinsic carrier density, different means to increase the carrier density in organic semiconductors can be applied:

1. (electro-)chemical doping,

2. carrier injection from contacts,

3. photo-generation of carriers, and

4. field-effect doping.

In the following section these methods will be briefly discussed together with their realization in different device structures.

\subsection{Device Structures and Properties}

Controlled doping has been one of the keys for the success of semiconductor microelectronics. There have been efforts to use tools like ion implantation doping also for organic semiconductors [37], however, due to the concomitant ion beam damages and the need for sophisticated equipment this method is probably not compatible with organic devices. Other techniques, like chemical doping by adding strong electron donors or acceptors as well as by electrochemical means have been successfully applied [38,39]. At this point one should also mention that often unintentional doping of organic materials already occurs during the synthesis or handling of the materials since in many cases ambient oxygen causes p-type doping of organic materials. Thus at present, controlled doping in organic semiconductors is still in its infancy and needs further investigations to employ it as a powerful tool for organic electronics. 


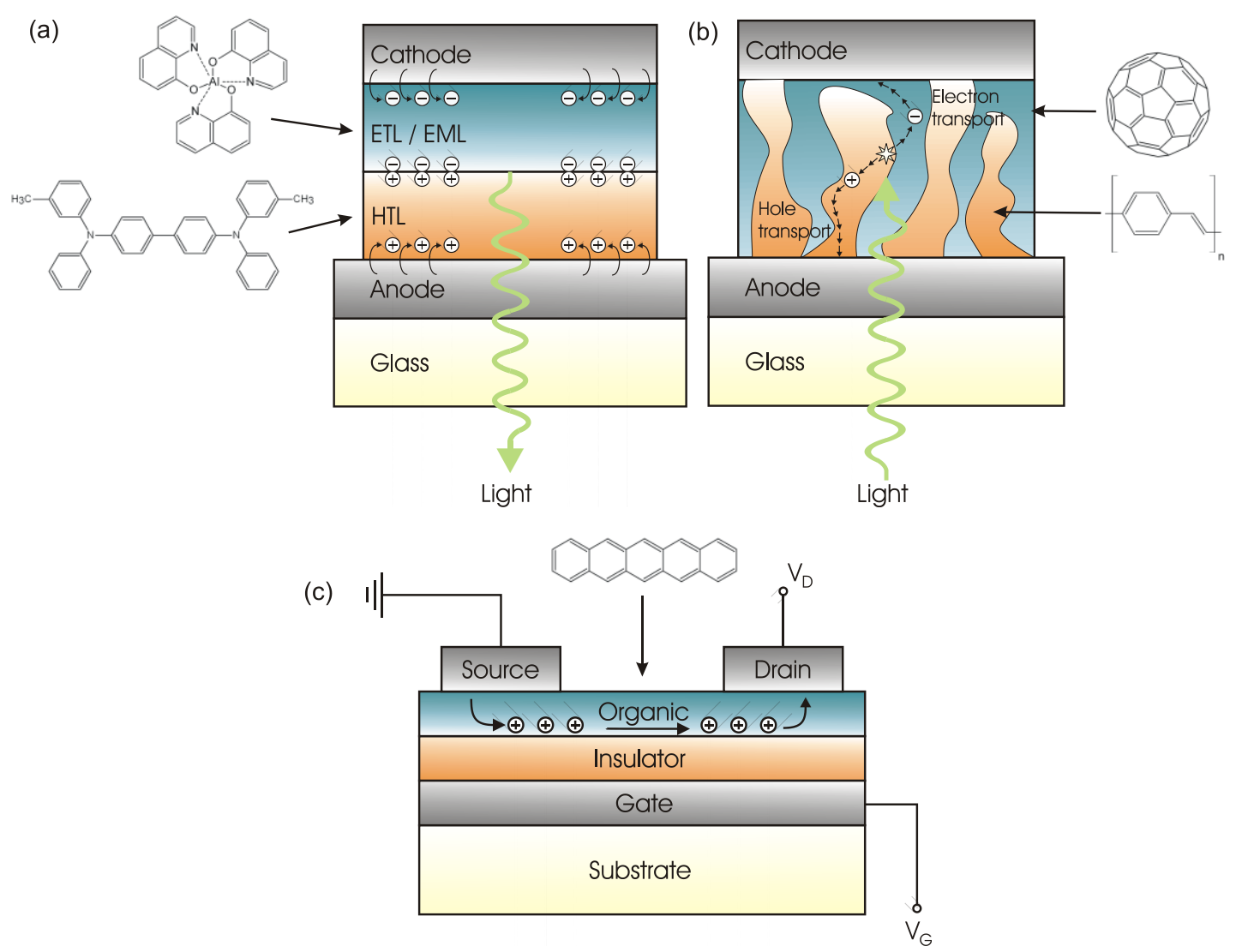

Fig. 8 Different types of organic semiconductor devices are shown. (a) Organic light-emitting diode (OLED): Typically, a heterolayer structure is used, where HTL stands for hole transport layer and ETL for electron transport layer, EML denotes the emission layer. Instead of the displayed combination of a triphenylamine derivative and $\mathrm{Alq}_{3}$, polymeric OLEDs usually employ a conductive polymer (PEDOT:PSS) together with luminescent polymers like PPV or PFO derivatives. (b) Organic photovoltaic cell (OPVC): The so-called bulk-heterojunction devices usually consist of a mixture of soluble PPV (or P3AT) and fullerene derivatives. Alternatively, mixed layers of evaporated small molecules like $\mathrm{CuPc}$ and $\mathrm{C}_{60}$ can be used. (c) Organic field-effect transistor (OFET): Prototypical materials in p-channel OFETs are pentacene as a low molecular weight material and P3AT as a conjugated polymer, respectively. Among others, e.g. $\mathrm{C}_{60}$ can be employed in n-channel transistors.

Injection of charge carriers from contacts is essentially the process that governs device operation in organic light-emitting devices (OLEDs) (see Fig. 8a). This requires low energetic barriers at the metalorganic interfaces for both contacts to inject equally high amounts of electrons and holes, which is required for a balanced charge carrier flow. Thus the interface energetic structure plays a very crucial role for achieving efficient OLEDs. Another process that comes into play is space-charge limitation of the current. Due to relatively high electric fields being applied to OLEDs (typically 5 to $10 \mathrm{~V}$ across a layer thickness of $100 \mathrm{~nm}$ yield $F=0.5 \ldots 1 \mathrm{MV} / \mathrm{cm}$ ) materials with low mobility such as $\mathrm{Alq}_{3}$ (having an electron mobility of $10^{-5} \mathrm{~cm}^{2} / \mathrm{Vs}$ ) still yield high enough current densities for display applications. This is a consequence of the space-charge limited current scaling with the $3^{\text {rd }}$ power of the reciprocal thickness [40]:

$$
j_{S C L C}=\frac{9}{8} \varepsilon \varepsilon_{0} \mu \frac{V^{2}}{d^{3}}
$$


Apart from charge carrier transport, the efficiency of OLEDs is also strongly influenced by photophysical processes. First of all, materials with a high fluorescence quantum yield are required. However, since a large fraction of the excited states formed by charge carrier recombination are triplets, the most efficient OLEDs nowadays make use of energy transfer to so-called triplet emitters, where the presence of heavy metals renders the transition from the triplet state to the ground state via phosphorescence an allowed process [41].

The second important device application of organic semiconductors is in organic photovoltaic cells (OPVCs) (see Fig. 8b). In spite of their high absorption coefficient, which exceeds $10^{5} \mathrm{~cm}^{-1}$ in most materials, the application of organic semiconductors in OPVCs faces the problem of the large exciton binding energy which prohibits efficient exciton dissociation. This can be overcome by making use of a photoinduced charge transfer between an electron donor like PPV and the fullerene $\mathrm{C}_{60}$ as an acceptor [42]. Due to the short exciton diffusion length of typically $10 \mathrm{~nm}$ only, efficient OPVCs use the so-called bulk-heterojunction concept of mixing donor and acceptor in one single layer. In spite of the huge progress recently achieved, there are still challenges to achieve sufficient lifetime of OPVCs under ambient conditions or the availability of low-band gap materials to make better use of the solar spectrum $[43,44]$. Organic field-effect transistors (OFETs) (see Fig. 8c) are 3-terminal devices in which the charge carrier density in the channel between source and drain contacts can be controlled by the applied gate voltage across a thin dielectric [45]. The current is then given by

$$
I_{d}=\frac{W}{L} C_{i} \mu\left(V_{g}-V_{t}\right) V_{d}
$$

in the linear region, and by

$$
I_{d}=\frac{W}{2 L} C_{i} \mu\left(V_{g}-V_{t}\right)^{2}
$$

in the saturation regime. Thus the performance of OFETs can be tuned to some degree by using suitable geometries with short channel length $L$ or thin insulating layers (leading to higher values for $C_{i}$ ), but it is clear that also the mobility needs to be high (in the range of amorphous $\mathrm{Si}$ ) to realize switching at frequencies higher than about $100 \mathrm{kHz}$ which will be needed for more demanding applications in the future $[46,47]$. This requires materials and methods to grow highly ordered organic semiconductor films. A further challenge will be to realize CMOS-like organic integrated circuits by using materials with ambipolar charge transport properties.

\section{Outline of this Book}

The following chapters are intended to give an up-to-date overview of the different facets of the subject of this book. They are based in articles published previously in a special issue of physica status solidi (a) (Vol. 201 (6), May 2004) devoted to this topic. In the first chapter on Growth and Interfaces, F. Schreiber describes organic molecular beam deposition with emphasis on growth studies beyond the first monolayer, i.e. the evolution of the film structure and morphology. The following two contributions by M. Knupfer and H. Peisert and by H. Ishii et al. deal with the electronic structure at interfaces between organic semiconductors and metals. In particular, issues like energy level alignment, formation of interface dipoles, band bending as well as interactions and molecular orientation at interfaces are studied. In the next article, M. Cölle and W. Brütting present thermal and structural investigations on the isomerism of the $\mathrm{Alq}_{3}$ molecule together with the first direct observation of the triplet state in this prototype organic electroluminescent material.

The latter subject directly leads to next chapter, which is devoted to the Photophysics of organic semiconductors. In the first article, G. Lanzani et al. give an overview on the contributions of ultrafast spectroscopy to the understanding of photophysics in conjugated polymers. S. Gamerith et al. investigate the 
emission properties of polyfluorene type polymers and their implications for polymer light-emitting diodes. V.I. Arkhipov and H. Bässler present experimental and theoretical aspects on the problem of exciton dissociation and charge photogeneration in conjugated polymers. The final two articles of this block deal with the problem of the singlet-triplet ratio in OLEDs: M. Wohlgenannt presents investigations on polarons in $\pi$-conjugated polymers and oligomers providing strong evidence for spin-dependent recombination, while $\mathrm{M}$. Baldo and $\mathrm{M}$. Segal study exciton formation and energy transfer processes using phosphorescent molecular materials which are involved in highly efficient phosphorescent OLEDs. The third big chapter of this book is dealing with Transport and Devices. The topic is introduced by an article by R. Schmechel and H. von Seggern on electronic traps in organic semiconductors, which is a key issue for understanding and optimizing charge transport in organic devices. In the following paper, C. Tanase et al. elucidate the fundamental differences in charge carrier transport between OLEDs and OFETs by studying the dependence of the charge carrier mobility on carrier density. The next two articles deal with modelling of organic devices: First Y. Roichman et al. present a self consistent picture of charge injection and transport in low mobility disordered organic semiconductors. Then S. Scheinert and G. Paasch nicely demonstrate the strength of a combination of device design and fabrication, experimental data analysis and numerical simulation for understanding and improving OFETs. High mobility organic single-crystal field-effect transistors are studied by R.W.I. de Boer et al. Their investigations show that single-crystal OFETs are ideal model systems for the study of intrinsic electronic transport properties of organic molecular semiconductors. The following paper by I. Riedel and V. Dyakonov gives an overview of the state-of-the-art in bulk heterojunction photovoltaic devices based on polymerfullerene blend systems. Device applications of organic semiconductors in OLEDs are the subject of the remaining three articles: First, M.M. de Kok et al. report on the importance of the hole injection layer for efficiency and lifetime of polymer LEDs. Then D. Berner et al. provide a combined experimental study together with modelling to understand organic multi-layer OLEDs. Finally, W. Rieß et al. give an impressive demonstration of how combinatorial device optimization has lead to the development of a prototype 20-inch active-matrix full-colour OLED display driven by amorphous silicon thin-film transistors.

Acknowledgements I would like to thank all contributing authors of this book for their efforts to present their work on the various aspects of this topic. Furthermore, I thank the editorial office of Wiley-VCH, in particular Ron Schulz, for their cooperation to publish this book.

\section{References}

1. N. Karl, Organic Semiconductors, in O. Madelung, M. Schulz, and H. Weiss (Eds.), Landolt-Boernstein (New Series), Group III, Vol. 17 Semicondcuctors, Subvolume 17i, page 106. Springer, Berlin, 1985.

2. J. Koenigsberger, K. Schilling, Ann. Physik 32, 179 (1910).

3. M. Volmer, Ann. Physik 40, 775 (1913).

4. M. Pope, H. Kallmann, and P. Magnante, J. Chem. Phys. 38, 2042 (1963).

5. W. Helfrich and W.G. Schneider, Phys. Rev. Lett. 140, 229 (1965).

6. E.A. Silinsh. Organic Molecular Crystals. Springer, Berlin 1980.

7. M. Pope and C.E. Swenberg. Electronic Processes in Organic Crystals. Clarendon Press, Oxford 1982.

8. D.F. Williams and M. Schadt, Proc. IEEE (Lett.) 58, 476 (1970).

9. C. K. Chiang, C. R. Fincher, Jr., Y. W. Park, A. J. Heeger, H. Shirakawa, E. J. Louis, S. C. Gau, and Alan G. MacDiarmid, Phys. Rev. Lett. 39, 1098 (1977).

10. T.A. Skotheim (Ed.). Handbook of Conducting Polymers. M. Dekker, New York 1986.

11. P. M. Borsenberger and D.S. Weiss. Organic Photoreceptors for Imaging Systems. M. Dekker, New York 1993.

12. C.W. Tang, Appl. Phys. Lett. 48, 183 (1986).

13. H. Koezuka, A. Tsumara, and T. Ando, Synth. Met. 18, 699 (1987).

14. J.H. Burroughes, C.A. Jones, and R.H. Friend, Nature 335, 137 (1988).

15. G. Horowitz, D. Fichou, X.Z. Peng, Z. Xu, and F. Garnier, Solid State Communications 72, 381 (1989).

16. C.W. Tang and S.A. VanSlyke, Appl. Phys. Lett. 51, 913 (1987).

17. C.W. Tang and S.A. VanSlyke, J. Appl. Phys. 65, 3610 (1989). 
18. J.H. Burroughes, D.D.C. Bradley, A.R. Brown, R.N. Marks, K. Mackay, R.H. Friend, P.L. Burn, and A.B. Holmes, Nature 347, 539 (1990).

19. D. Braun and A.J. Heeger, Appl. Phys. Lett. 58, 1982 (1991).

20. Pioneer Co. (Japan). In November 1997 Pioneer Co. in Japan commercialized a monochrome 256x64 dot matrix OLED display for automotive applications.

21. Materials Today, September 2004: C. Reese et al. p. 20-27; I.D.W. Samuel et al. p. 28-35; N.S. Sariciftci p. 36-40; J.K. Borchardt p. $42-46$ (www.materialstoday.com).

22. K.P.C. Vollhardt, Organische Chemie, VCH-Verlag, Weinheim 1990

23. M. Pope and C.E. Swenberg. Electronic Processes in Organic Crystals and Polymers. Oxford University Press, Oxford 1999.

24. R. Farchioni, G. Grosso (Eds.), Organic Electronic Materials, Springer 2001.

25. F. Faupel, C. Dimitrakopoulos, A. Kahn, C. Wöll (Eds.), Organic Electronics, Special Issue of J. Mater. Res. 19 (7), 2004.

26. H. Bässler, phys. stat. sol. (b) 107, 9 (1981)

27. N. Karl, J. Marktanner, Mol. Cryst. Liq. Cryst. 355, 149 (2001).

28. N. Karl: Charge Carrier Mobility in Organic Crystals, in R. Farchioni, G. Grosso (Eds.), Organic Electronic Materials, Springer 2001.

29. W. Brütting, S. Berleb, A. Mückl, Organic Electronics 2, 1, 1(2001).

30. J.C. Scott, J. Vac. Sci. Technol. A 21, 521 (2003).

31. U. Wolf, V.I. Arkhipov, H. Bässler, Phys. Rev. B 59, 7507 (1999); ibid. 59, 7514 (2001).

32. P.W.M. Blom, M.C.J.M. Vissenberg, Mater. Sci. Eng. 27, 53 (2000).

33. M.A. Baldo, S.R. Forrest, Phys. Rev. B 64, 085201 (2001).

34. C.D. Dimitrakopoulos, P.R.L. Malenfant. Adv. Mater. 14, 99 (2002).

35. H. Klauk, M. Halik, U. Zschieschang, G. Schmid, C. Dehm, Appl. Phys. Lett. 84, 2673 (2004).

36. T.W. Kelley, D.V. Muyers, P.F. Baude, T.P. Smith, T.D. Jones, Mat. Res. Soc. Symp. Proc. 771, 169 (2003).

37. A. Moliton. Ion implantation doping of electroactive polymers and device fabrication. In J.R. Reynolds T.A. Skotheim, and R.L. Elsenbaumer (Eds.), Handbook of Conducting Polymers. Marcel Dekker, New York 1998.

38. M. Pfeiffer, K. Leo, X. Zhou, J.S. Huang, M. Hofmann, A. Werner, J. Blochwitz-Nimoth, Organic Electronics 4, 89 (2003)

39. M. Gross, D.C. Müller, H.-G. Nothofer, U. Scherf, D. Neher, C. Bräuchle, K. Meerholz, Nature 405, 661 (2000).

40. M.A. Lampert and P. Mark, Current Injection in Solids, Academic Press, New York 1970.

41. M.A. Baldo, D.F. O’brien, Y. You, A. Shoustikov, S. Sibley, M.E. Thompson, S.R. Forrest, Nature 395, 151 (1998); S.R. Forrest, Organic Electronics 4, 45 (2003).

42. N.S. Sariciftci, L. Smilowitz, A.J. Heeger, F. Wudl, Science 258, 1474 (1992).

43. C.J. Brabec, N.S. Sariciftci, J.C. Hummelen, Adv. Funct. Mater. 11, 15 (2001).

44. P. Peumans, S. Uchida, S.R. Forrest, Nature 425, 158 (2003).

45. G. Horowitz, Adv. Mater. 10, 365 (1998).

46. G.H. Gelinck, et al. Nature Mater. 3, 106 (2004).

47. A. Knobloch, A. Manuelli, A. Bernds, W. Clemens, J. Appl. Phys. 96, 2286 (2004). 